\title{
Diferencias entre hombres y mujeres en el automanejo de la diabetes mellitus tipo 2: estudio cualitativo
}

\author{
Differences among men and women in the self-management of Type 2 Diabetes Mellitus: qualitative study
}

\section{Objetivo}

Comprender las diferencias entre los hombres y las mujeres que viven con diabetes mellitus tipo 2 (DM2), las barreras y los desafíos en su autocontrol.

\section{Diseño, lugar y población}

Estudio cualitativo con cinco grupos focales (mixtos) y nueve entrevistas individuales. Se incluyeron 35 participantes entre hombres y mujeres, con DM2, que fueron reclutados en un centro de educación en diabetes en Toronto, Canadá.

\section{Resultados}

La edad promedio de los participantes fue de 57 años y el $51,4 \%$ de los entrevistados fueron mujeres. Realizaron un análisis temático del material recolectado en las entrevistas que permitió identificar cinco tópicos:1) la revelación y su identificación como una persona que vive con diabetes; 2) automonitorización de la glucosa en la sangre; 3) dieta en diversos contextos; 4) utilización de los recursos existentes para la diabetes y 5) el apoyo social.

El estudio mostró que las mujeres daban a conocer su diabetes más fácilmente y la integraban a su vida cotidiana, mientras que los hombres eran más reacios a decir a amigos y familiares acerca de su enfermedad y se cuidaban (prácticas de autocuidado) menos en los entornos
Mathew R, y col. BMC Family Practice 2012;13:122. sociales. Los hombres se centraban en los aspectos prácticos del automonitoreo de la glucemia experimentando con diversos dispositivos en la gestión de la diabetes para reducir la dependencia a los medicamentos; mientras que las mujeres se centraban en los componentes afectivos del automonitoreo de la glucemia. Las mujeres hablaron de alimentos restringidos, de sus dietas percibidas como prohibidas, mientras que muchos hombres dijeron moderar el consumo de alimentos percibidos como poco saludables, excepto en situaciones sociales. Las mujeres utilizaban los recursos socialmente interactivos (clases de educación y grupos de apoyo), mientras que los hombres confíaban más en el aprendizaje autodirigido. Finalmente, los hombres y las mujeres informaron que querían apoyo médico, tanto afectivo como en la práctica (atención) respecto a los aspectos de la autogestión de la enfermedad.

\section{Conclusiones}

Los resultados destacan las diferencias en las necesidades y los retos en el autocontrol de la diabetes entre los hombres y las mujeres, tales como: informar el diagnóstico de diabetes, la atención, el asesoramiento $y$ el apoyo con enfoque de género.

Fuente de financiamiento/conflicto de interés: Universidad Ryerson. Los autores no refieren conflicto de interés.

\section{Comentario}

En la literatura se han reportado diferencias en el manejo de la diabetes entre hombres y mujeres, en particular en sus creencias, actitudes, temores y preocupaciones acerca de la enfermedad. Las mujeres, más que los hombres, ven a la diabetes como algo negativo que afecta sus vidas. Ante el diagnóstico, más mujeres reportan pérdida de la salud relacionada con la diabetes y la mortalidad temprana en comparación con los hombres'. Por ejemplo, las mujeres se preocupan más por las complicaciones agudas de la diabetes, tales como hipoglucemia, y las complicaciones crónicas, como enfermedad cardiovascular y renal ${ }^{1,2}$. Mientras que los hombres están más preocupados de que la diabetes limite sus estilos de vida, pero además piensan que es controlable ${ }^{2,3}$. También hay literatura que demuestra diferencias de género en las barreras en cuanto a la libre gestión de la DM2 $2^{4,5,6}$. Un estudio realizado por Whittemore y col. sugiere que las barreras respecto al autocuidado entre las mujeres podrían incluir la falta de confianza en sí mismas y un apoyo inadecuado de su entorno inmediato, amigos y familia. Cherrington y col. indagaron acerca de las barreras y los facilitadores para el autocuidado de la diabetes entre los inmigrantes latinos encontrando que en las mujeres las barreras están relacionadas con un apoyo social inadecuado en el hogar, especialmente en lo que atañe a la preparación de alimentos. Mientras que en los hombres las barreras predominantes son la falta de flexibilidad horaria para comer en los lugares de trabajo?.

\section{Conclusiones de la comentadora}

A pesar de disponer de información que describe las diferencias de género respecto a vivir con DM2, aún hay una brecha que falta transitar. Apoyarnos en otros marcos teóricos como el enfoque relacional ${ }^{8}$ y las representaciones sociales ${ }^{9,10}$ nos brindarían una mirada más profunda respecto a ¿qué significa para los hombres y para las mujeres vivir con una enfermedad crónica como la diabetes? Dado que, el proceso salud/enfermedad/atención compromete muchas esferas de la vida que van desde las biológicas, psicológicas, ideológicas, culturales, hasta las políticas e institucionales, particulares de cada uno y de cada lugar; una mejor comprensión facilitaría la promoción de estrategias directas para mejorar la atención y la gestión de apoyo a las personas que viven con DM2.

\section{Nanci Giraudo [ Servicio de Medicina Familiar y Comunitaria del Hospital Italiano de Buenos Aires. nanci.giraudo@hospitalitaliano.org.ar]}

Giraudo N. Diferencias entre hombres y mujeres en el automanejo de la Diabetes Mellitus Tipo 2: estudio cualitativo. Evid Actual Pract Amb. 18(3):71. Jul-Sep. Comentado de: Mathew R, y col. Self-management experiences among men and women with type 2 diabetes mellitus: a qualitative analysis. BMC Family Practice 2012;13:122. PMID:23249410

\section{Referencias bibliográficas}

1. Dietrich UC. Factors influencing the attitudes held by women with type II diabetes: a qualitative study. Patient Educ Couns 1996;29(1):13-23.

2. Jonsson PM, Sterky G, Gafvels C, Ostman J: Gender equity in health care: the case of Swedish diabetes care. Health Care Women Int 2000, 21(5):413-431.

3. Brown SA, y col. Gender and treatment differences in knowledge, health beliefs, and metabolic control in Mexican Americans with type 2 diabetes. Diabetes Educ 2000;26(3):425-438.

4. Aljasem L, y col. The impact of barriers and self efficacy on self-care behaviors in type 2 diabetes. Diabetes Educ 2001;27(3):393-404.

5. Glasgow R, y col. Psychosocial barriers to diabetes self management and quality of life. Diabetes Spectrum 2001;41(1):33-41.

6. Nagelkerk J, y col. Perceived barriers and effective strategies to diabetes self-management. Journal of Adv Nurs 2006;54(2):151-158.

7. Cherrington A, y col. Developing a family-based diabetes program for Latino immigrants: Do Men and women face the same barriers? Fam Community Health 2011;34(4):280-290.

8. Menéndez E. Morir de alcohol: Saber y hegemonía médica. México: Alianza;1990.

9. Moscovici S. El psicoanálisis, su imagen y su público. Buenos Aires: Huemul; 1979

10. Jodelet D. La representación social: Fenómenos, conceptos y teoría. En: Moscovici S. Psicología Social Il. Barcelona, España: Paidós; 1985. 